\title{
Prilocaine for Bier's Block: how safe is safe?
}

\author{
K. BARTHOLOMEW \& J. P. SLOAN
}

Accident and Emergency Department, Leeds General Infirmary, Leeds

\section{SUMMARY}

Prilocaine has become the agent of choice for Bier's block (or intravenous regional anaesthesia - IVRA), since 1983 when the product licence of bupivacaine was withdrawn for this purpose owing to fatal or serious complications.

No serious complications have been documented in the literature relating to prilocaine in IVRA and we have conducted a survey within the U.K. which indicates that about 45000 Bier's blocks have been carried out with prilocaine without convulsion, arrhythmia or fatality. This includes cases of accidental cuff deflation or even failure to inflate the cuff, resulting in bolus doses to the circulation.

Prilocaine has now been in use since 1964 and the Committee for Safety of Medicines has no deaths on record over a 25-year period. (This includes other types of regional anaesthesia).

We suggest that intravenous regional anaesthesia using prilocaine is a safe technique. It is highly unlikely that fatalities will occur, provided present guidelines are adhered to. We can find no reason to limit its use to trained anaesthetists only, but would recommend that a strict protocol is adhered to.

\section{INTRODUCTION}

IVRA was first performed by August Karl Gustav Bier in 1908 using procaine. The technique is employed as an alternative to general anaesthesia and is most commonly used for procedures involving the upper limb. It is particularly useful for reduction and manipulation of fractures in patients presenting to accident and emergency departments.

Correspondence: Mr J. P. Sloan, Accident and Emergency Department, General Infirmary, Leeds LS1 3EX, U.K. 
The procedure was revived in the 1960's (Holmes, 1963) and again in the lat 1970's (Wallace \& Milne, 1978). There was considerable controversy about the agent of choice and bupivacaine became popular owing to its superior analgesi@ efficacy, muscle relaxant effects and supposed low toxicity in comparison with lignocaine.

In 1983 the product licence of bupivacaine was revoked for the purpose of IVR after several reports of toxic reactions leading to convulsions and death (5 case between 1979-1983 in the U.K.). The failure of automatic tourniquets was con $\overline{\mathrm{D}}$ tributory in some of these cases, but despite the Hazard warning HN (82)7 issued by the Department of Health and Social Security, 2 further fatalities did occur.

Prilocaine has since become the agent of choice. It has a much higher margin of safety than bupivacaine, and is also partially extracted by the pulmonary circulation $\vec{\omega}$ It is one of the amide group of local anaesthetics and is also available as preservative-free preparation. Both these factors considerably reduce the incidence of anaphylactic reactions. It was not favoured at one time because of the possible risk of methaemoglobinaemia, but it is now accepted that this is very unlikely te occur at the dose appropriate to IVRA.

There was an increasing consensus of opinion at the time of the bupivacaine deaths, particularly amongst anaesthetists, that this potentially dangerous procedure should be conducted by doctors skilled in resuscitaion. It was generally considere⿻্乛龰 that anaesthetists were likely to be the most experienced group in airway man 3 agement and to be most familiar with the administration of thiopentone o卬 alternative anticonvulsant agents.

There are surprising differences, however, between hospitals. A service is pto유 vided by the anaesthetic department in one hospital, and it is mandatory that क्वiv anaesthetist is present to administer the block, yet in another hospital in the same city it is routine for accident and emergency SHOs to carry out IVRA.

We set out to discover the extent to which Bier blocks are used, what the्ष complication rate is using prilocaine, and how protocol and policy vary betweer? hospitals.

\section{METHOD}

We devised and circulated a questionnaire with 11 questions to 106 accident and emergency departments in the United Kingdom. The selection criterion for the A\&E departments was an excess of 45000 new patients per year as listed in the 1988 A\&E Year Book.

The questions were directed towards usage of prilocaine for intravenous regionaE: anaesthesia, duration and extent of use, variations in procedure and occurrence and severity of complications. 


\section{RESULTS}

A total of $72(68 \%)$ of 106 questionnaires were returned. Sixty-five per cent of A\&E departments actually use Bier's blocks and 35\% do not (Fig. 1).

Of those performing Bier's blocks almost all $(96 \%)$ used prilocaine with the exception of two departments (4\%) who still used lignocaine (Fig. 2). None reported the use of any other local anaesthetic agent although several mentioned the increasing use of alternative techniques such as sedation with midazolam or haematoma block for reasons of time economy or unavailability of anaesthetic staff. Conversely a few commented that general anaesthesia was preferred and anaesthetic cover was not a problem.

The mean length of time prilocaine has been used for IVRA is 5 years, most departments ranging between two and seven years, and some as long as 10 and 15 years.

From the number of blocks each department estimated had been performed with prilocaine, the total number was calculated to be of the order of 45000 . (exact estimate $45707+/-2298)$.

\section{Complications}

No serious or fatal complications were reported by any of the 45 departments using Bier's blocks. Several minor complications were reported as follows (Fig. 3):Cuff problems (2 cases, neither causing a detectable clinical reaction); poor efficacy, especially in large men (commented on by 3 departments); petechial rash ( 1 case);

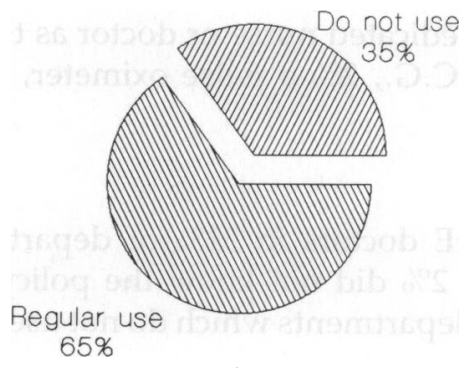

Fig. 1. Use of Bier's Block in A\&E (72 U.K. A\&E departments seeing $>45000$ patients).

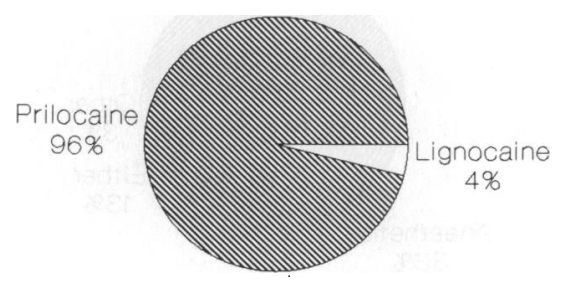

Fig. 2. Drug used. 


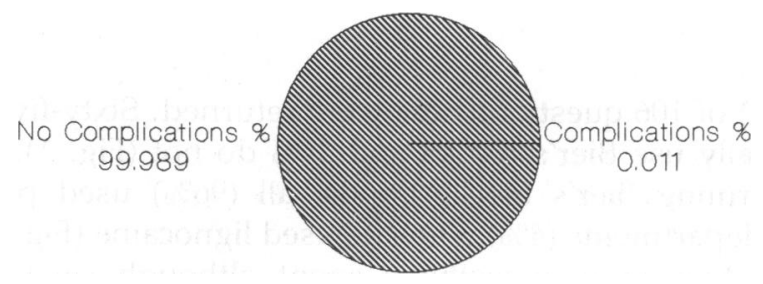

Fig. 3. Complications of 45000 cases using prilocaine.

and venous congestion ( 1 case). Despite specific mention of cuff failure no complications were reported as a consequence.

\section{Protocols}

There was a wide variety in procedures and guidelines between the 45 departments using Prilocaine. Thirty-four departments $(75 \%)$ have a maximum dose limit, $6(13 \%)$ do not have a stipulated limit and $5(11 \%)$ replied 'don't know'.

The maximum dose limit is $3 \mathrm{mg} / \mathrm{kg}$ in $76 \%$ of departments with a set limit. $\vec{c}$ Four other departments reported limits of $4 \mathrm{mg} / \mathrm{kg},(1), 5 \mathrm{mg} / \mathrm{kg},(1)$ and

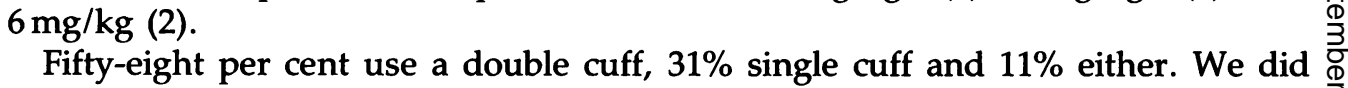
not collect data regarding the relative use of automated and manual cuffs.

\section{Monitoring}

Sixty-four per cent have a dedicated nurse or doctor as the sole form of monitoring. In addition, $13 \%$ use an E.C.G., $2 \%$ a pulse oximeter, and $4 \%$ both.

Who conducts the procedure?

IVRA is performed by A\&E doctors in $51 \%$ of departments, by anaesthetists in $33 \%$ and by either in $13 \%$. $2 \%$ did not know the policy (Fig. 4).

Sixty per cent (including departments which do not use Bier's blocks), considered it

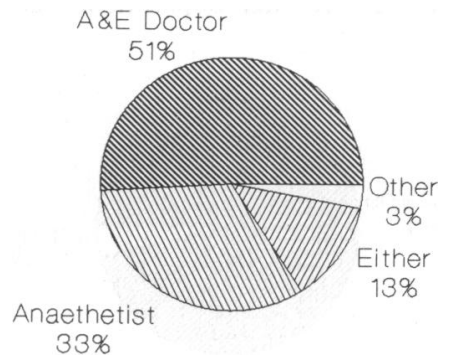

Fig. 4. Who performs it? 
safe for any appropriately trained and competent doctor to conduct IVRA with a trained nurse present and another doctor close at hand but not necessarily in the room.

Twenty-nine per cent considered that only anaesthetists should perform IVRA under the same conditions as above. Eleven per cent thought that two doctors should be present in the room for the duration of the procedure.

\section{DISCUSSION}

Prilocaine would appear to be a relatively safe local anaesthetic. This survey has demonstrated that no serious complications occurred in a series of about 45000 Bier's blocks using prilocaine. We have contacted Astra, the manufacturers of Citanest $(0.5 \%$ prilocaine, preservative-free) who know of no adverse reactions with the exception of one localised anaphylactoid reaction (Ruiz et al., 1987) caused by injecting the drug too rapidly, resulting in damage to the vasculature.

The Committee on Safety of Medicines data records from July 1963-July 1989 indicate that prilocaine has not caused any deaths over a 26-year period. This includes all modes of use, not just IVRA. Single constituent products (e.g. Citanest) have fewer reported reactions (48) than multi-constituent products (78). The latter include adrenaline-containing preparations and 'EMLA' local anaesthetic cream. It is, of course, the single-constituent preservative-free $0.5 \%$ Citanest which is marketed for and recommended for IVRA.

Accidental intravenous injections doses of prilocaine have occurred with doses intended for IVRA without complication. This has happened both as the result of early (accidental) tourniquet deflation (Robinson \& Shimmings, 1989) and also after unintentional failure to inflate the cuff at all (personal communication). The patients in both cases were totally unaware of the event and suffered no symptoms.

It is known that local anaesthetic leakage occurs into the systemic circulation during the period that the tourniquet is inflated. Despite proper use of the tourniquet and correct inflation pressure, there is escape via the intraosseous circulation and also through the deep veins due to the high venous pressures resulting from the injection of local anaesthetic. (El-Hasson et al., 1984). It has also been shown that fracture manipulation leads to increases in pressure which can cause leakage (Quinton et al., 1988).

Systemic prilocaine and lignocaine levels have been compared (Bader et al., 1988) after deflation of the tourniquet, and prilocaine levels were found to be significantly lower than lignocaine levels after administration of equal doses. This is thought to be due to greater uptake by peripheral tissues and slower release to the circulation following tourniquet deflation, and to the 'first pass' effect of extraction and metabolism of the pulmonary circulation.

Bupivacaine levels were also measured both during the phase of cuff inflation and after deflation (Mason, 1983). A pilot study of 5 patients resulted in toxic levels in two of the subjects after $5 \mathrm{~min}$ despite correct inflation of the cuff. Therefore the trial was considered dangerous and abandoned. Further evidence of 
the toxicity of bupivacaine is that the incidence of convulsions while bupivacaine ${ }_{\bar{\Phi}}$ was actually in use was estimated to be between 2 and 7 in 10000 (Health, 1982).

This study has shown that there is undoubtedly room for improvements in the design and display of protocols. One method is to use a wall chart (Quinton, 1989). $\stackrel{.}{\rightarrow}$ This ensures that all staff are familiar with the general procedure and also would $\stackrel{0}{\circ}$ provide a constant reminder of the most important points. These are the maximum? recommended dose and the mandatory time interval for which the cuff must remain inflated following injection of prilocaine.

Certain criteria must be satisfied before IVRA is performed; all equipment must be properly maintained, regularly serviced and checked before use. This includes both the tourniquet or cuff apparatus and the resuscitation equipment. The latter: should include facilities for maintenance of the airway and ventilation of the lungs $\vec{\omega}$ with oxygen. The immediate availability of anticonvulsant agents such as thiopen- $\frac{\curvearrowright}{\Phi}$ tone, diazepam and midazolam should be established beforehand. The doctor who performs the procedure should possess a good working knowledge of the possible ${ }_{\omega}$ problems and must be skilled in resuscitation. We suggest that there must be $a \vec{\infty}_{\infty}$ competent assistant e.g. experienced sister or staff nurse dedicated to the procedure and a second doctor immediately available but not necessarily present in the $\stackrel{?}{?}$ room.

There are several accepted exclusion criteria for patients; the absolute contra- $-\frac{\pi}{0}$ indications are sickle cell disease, cellulitis and vascular disease. Hypertension $\stackrel{\mathbb{D}}{3}$ and children aged less than 10 years of age are relative contra-indications. The patients must be able to give informed consent and be fully cooperative. Margy $\overrightarrow{0}$ would feel that the procedure is best avoided in all children.

There is obviously a greater potential for error with a double cuff tourniquềt and we suggest that it is not necessary for the brief duration blocks $(20-30 \mathrm{~min})$ ? which are usually required in accident and emergency. A single cuff is less likely to give rise to confusion.

There are certain points that should be emphasized on charted guidelines or $\stackrel{\varnothing}{\circ}$ protocols: - venous access to the systemic circulation must always be secured (by $\vec{\partial}$ a second cannula in the contralateral limb) before the injection of the local anaesthetic via the other cannula distal to the cuff. The limb should be exsanguinated, either by elevation for $3 \mathrm{~min}$ with compression of the brachial artery or by Esmarch bandage, (usually too painful if a fracture is present) and the cuff then inflated to 3 . at least $100 \mathrm{~mm} \mathrm{Hg}$ above systolic pressure in the arm and $200 \mathrm{~mm} \mathrm{Hg}$ above in the leg. It is always worthwhile checking that the cuff is actually inflated and the radial pulse is no longer palpable, prilocaine can then be given by slow injection $₹$ (over several $\mathrm{min}$ ). The recommended safe dose is $3 \mathrm{mg} / \mathrm{kg}$, which is equivalent to $40 \mathrm{ml}(0.5 \%$ solution for a $67 \mathrm{Kg}$ adult, i.e. $200 \mathrm{mg}$. The cuff should remain inflated for at least $20 \mathrm{~min}$ from the time of injection and should then be deflated slowly or deflated for a few seconds and then reinflated for a minute whilst the patient is $\tilde{\sigma}$ observed for symptoms of toxicity. The classical signs of local anaesthetic toxicity $N$ are unease, perioral tingling, dizziness and slurring of speech which progress to స్心 loss of consciousness and convulsions (although the two latter are not recorded tog have occurred after prilocaine).

We did not collect any data on starvation policy in the survey. It is commonly 
recommended that patients should be starved for $4 \mathrm{~h}$ before IVRA but we are aware of many A\&E departments in which blocks are performed on unstarved subjects with no adverse effects. It is preferable as an A\&E procedure to be able to perform manipulation and reduction as soon as possible, ideally as people come in off the street, not necessarily starved. This raises the question, do we actually need to starve patients before IVRA, since the risk of aspiration is negligible. It is already known that trauma delays gastric emptying and it is questionable whether a period of starvation is effective: - it is a matter of debate even before general anaesthesia. However, at the present time it would be unwise to recommend any policy which advocates the abandonment of starvation.

\section{REFERENCES}

Bader A., Concepcion M., Hurley R. J. \& Arthur G. R. (1988) Comparison of lidocaine and prilocaine for intravenous regional anaesthesia. Anaesthesiology 69, 409.

El-Hassan K. M., Hutton P. \& Black A. M. S. (1984) Venous pressures and arm volume changes during simulated Bier's block. Anaesthesia 39, 229.

Heath M. L. (1982) Deaths after intravenous regional anaesthesia. British Medical Journal 285, 913.

Holmes C. M. C. K. (1963) Intravenous Regional Anaesthesia. A useful method of producing anaesthesia of the limbs. Lancet, 245.

Mason M. A. (1983) Local anaesthesia with bupivacaine. Lancet, 1085.

Quinton D. N. (1989) Regional anaesthesia for hand surgery in Accident and Emergency. Hospital Update, 827.

Quinton D. N., Hughes J., Mace P. F. K. \& Aitkenhead A. R. (1988) Prilocaine leakage during tourniquet inflation in intravenous regional anaesthesia. The influence of fracture manipulation. Injury 19, 239.

Robinson D. A. \& Shimmings K. I. (1989) Uncomplicated accidental early tourniquet deflation during intravenous regional anaesthesia with prilocaine. Anaesthesia 44, 83.

Ruiz K., Stevens J. D. Train J. J. A. \& Watkins J. (1987) Anaphylactoid reactions to prilocaine. Anaesthesia 42, 1078.

Wallace W. A. \& Milne D. D. (1978) Intravenous regional anaesthesia. Hospital Update, 137. 